\title{
Standard Delay Eyeblink Classical Conditioning Is Independent of Awareness
}

\author{
Joseph R. Manns and Robert E. Clark \\ University of California, San Diego
}

\author{
Larry R. Squire \\ Veteran Affairs Healthcare System, San Diego, \\ and University of California, San Diego
}

\begin{abstract}
P. F. Lovibond and D. R. Shanks (2002) suggested that all forms of classical conditioning depend on awareness of the stimulus contingencies. This article considers the available data for eyeblink classical conditioning, including data from 2 studies (R. E. Clark, J. R. Manns, \& L. R. Squire, 2001; J. R. Manns, R. E. Clark, \& L. R. Squire, 2001) that were completed too recently to have been considered in their review. In addition, in response to questions raised by P. F. Lovibond and D. R. Shanks, 2 new analyses of data are presented from studies published previously. The available data from humans and experimental animals provide strong evidence that delay eyeblink classical conditioning (but not trace eyeblink classical conditioning) can be acquired and retained independently of the forebrain and independently of awareness. This conclusion applies to standard conditioning paradigms; for example, to single-cue delay conditioning when a tone is used as the conditioned stimulus (CS) and to differential delay conditioning when the positive and negative conditioned stimuli $(\mathrm{CS}+$ and $\mathrm{CS}-$ ) are a tone and white noise.
\end{abstract}

In a review of classical conditioning that focused on human studies from 1990 to 2001, Lovibond and Shanks (2002) suggested that all forms of classical conditioning are likely mediated by higher order cognitive processes. In humans, they proposed, this dependence on cognitive activity is manifested as a link between awareness of the stimulus contingencies and as conditioned responses (CRs). Thus, the authors suggested that awareness is necessary for conditioned performance and that no convincing examples of independence between awareness and classical conditioning have been reported.

One kind of classical conditioning considered in the review was eyeblink classical conditioning. There are two fundamentally different forms of eyeblink conditioning. In trace eyeblink conditioning, a short, empty interval separates the conditioned stimulus (CS) and the unconditioned stimulus (US). In delay eyeblink conditioning, the CS and US overlap and coterminate. Trace eyeblink conditioning shares several features with declarative memory, most notably the fact that learning depends on the hippocampus (Clark \& Squire, 1998; McGlinchey-Berroth, Carrillo, Gabrieli, Brawn, \& Disterhoft, 1997) and on awareness of the stimulus contingencies (Clark \& Squire, 1998; Manns, Clark, \& Squire,

Joseph R. Manns, Department of Psychology, University of California, San Diego; Robert E. Clark, Department of Psychiatry, University of California, San Diego; Larry R. Squire, Veteran Affairs Healthcare System, San Diego, and Departments of Psychiatry, Nuerosciences, and Psychology, University of California, San Diego.

This research was supported by the Medical Research Service of the Department of Veterans Affairs, National Institute of Mental Health Grant 24600, and the Metropolitan Life Foundation. We thank Craig Stark for assistance.

Correspondence concerning this article should be addressed to Larry R. Squire, Department of Veterans Affairs (116A), 3350 La Jolla Village Drive, San Diego, California 92161. E-mail: 1squire@ucsd.edu 2000a, 2000b). In contrast, delay eyeblink classical conditioning appears to be a quintessential example of nondeclarative memory.

Here, we consider the available data from studies of eyeblink classical conditioning in humans and experimental animals. Compelling evidence exists that delay eyeblink classical conditioning can be supported by lower level brain systems and that it can be acquired independently of awareness of the stimulus contingencies.

\section{Studies in Experimental Animals}

Standard delay eyeblink classical conditioning is perhaps the best understood example of associative learning in vertebrates. Many years of research in experimental animals have elucidated the crucial brain structures involved in acquisition, storage, and expression of the classically conditioned eyeblink response (for a review, see Thompson \& Krupa, 1994). The memory trace for classical delay eyeblink conditioning is formed and stored in the cerebellum. Data from conventional lesion studies, reversible lesion studies, electrical recording studies, and electrical stimulation studies indicate that the cerebellum is both necessary and sufficient to support delay eyeblink conditioning. For example, a small lesion $\left(1 \mathrm{~mm}^{3}\right)$ of the interpositus nucleus, one of the deep cerebellar nuclei, permanently abolishes the ability to exhibit any acquisition of the conditioned response while leaving the unconditioned response completely intact (Steinmetz, Lavond, Ivkovich, Logan, \& Thompson, 1992).

In contrast to the profound effects of cerebellar lesions, bilateral hippocampal lesions have no effect on delay eyeblink conditioning (Berger \& Orr, 1983; Schmaltz \& Theios, 1972; Solomon \& Moore, 1975). Indeed, decerebrate rabbits with no remaining forebrain tissue (i.e., removal of cerebral cortex, basal ganglia, limbic system, thalamus, and hypothalamus) exhibited normal retention of classically conditioned eyeblink responses using the delay pro- 
cedure (Mauk \& Thompson, 1987). Thus, the structures normally used to acquire the conditioned response must lie at or below the level of the midbrain. Further, cats with bilateral removal of cerebral cortex, basal ganglia, and limbic system ("hemispherectomized" cats) were able to acquire delay classical conditioning as well as intact animals (Norman, Villablanca, Brown, Schwafel, \& Buchwald, 1974). Because forebrain structures are not necessary for acquisition or retention of the classically conditioned eyeblink response using the delay procedure, it seems reasonable to suppose that such conditioning proceeds relatively automatically and presumably in the absence of awareness about the stimulus contingencies. Of course, direct information about awareness can be obtained only from humans.

\section{Studies in Humans}

Findings from studies of eyeblink classical conditioning in humans have been remarkably consistent with the findings from studies in experimental animals. Lesions of the cerebellum severely impaired acquisition of delay eyeblink conditioning (Daum, Schugens, et al., 1993; Topka, Valls-Solé, Massaquoi, \& Hallett, 1993). In contrast, bilateral lesions of the hippocampal formation spared the acquisition of delay eyeblink conditioning (Clark \& Squire, 1998; Gabrieli et al., 1995). Still, what is the evidence concerning the importance of awareness during delay conditioning?

In one series of studies, 20 participants were given either standard single-cue delay eyeblink conditioning $(1,000-\mathrm{Hz}$ tone $\mathrm{CS}$, airpuff US) or were given single-cue trace eyeblink conditioning (Manns et al., 2000a; Manns, Clark, \& Squire, 2001). Participants were also given seven true or false questions about the relationship between the CS and the US after 10 trials, after 60 trials, and after all 120 trials. Because the results for delay conditioning were obtained too recently to have been reviewed by Lovibond and Shanks (2002), we show the main findings in Figure 1. For the participants given trace conditioning, the awareness score after the first 10 conditioning trials predicted the overall level of conditioning across the 120 -trial session $(r=+.49, p<.05)$. In contrast, for the participants given delay conditioning, there was no relationship between the awareness score and conditioning $(r=-.13, p>$ .10). Moreover, for the participants given trace conditioning, the correlation between the awareness score and the conditioning score (total percent CRs) was significantly greater than the correlation for the participants given delay conditioning $(p<.05)$.

Another way of making the same point is to note that in the delay conditioning group, the 6 participants who correctly answered all seven true or false questions conditioned no better across the 120-trial session than the 14 participants who answered fewer than seven questions correctly; (mean number of correct answers $=4.0$, which was no better than the chance score of 3.5); $t(13)<1.0$. Those who answered all seven questions correctly emitted $40.7 \pm 8.4 \%$ CRs. Those who answered fewer questions correctly emitted $41.8 \pm 3.0 \%$ CRs, $t(18)<1.0$. Further, the 15 participants who answered all seven questions correctly at the end of the conditioning session (after all 120 trials) conditioned no better across the session than the 5 remaining participants who answered fewer questions correctly (mean number of correct answers $=4.4 ; 41.5 \pm 0.4 \%$ CRs vs. $41.2 \pm 0.4 \%$ CRs). The results were strikingly different for single-cue trace conditioning. The 6 participants who answered all seven questions correctly after the first 10 conditioning trials emitted more CRs (47.4 \pm 9.7\%) across the 120-trial session than did the other 14 participants (mean number of correct answers $=4.9 ; 28.3 \pm 3.4 \% \mathrm{CRs}), t(18)=2.36$, $p<.05$.

These studies are to our knowledge the first to compare directly the relationship between conditioning and awareness (knowledge about the stimulus contingencies) for both standard (single-cue) delay eyeblink conditioning and standard (single-cue) trace eyeblink conditioning. The finding with delay conditioning (Manns et al., 2001) used a method for assessing awareness that appears to satisfy the concerns raised by Lovibond and Shanks (2002). Thus, in the context of discussing our parallel study of trace conditioning (Manns et al., 2000a), they pointed out that the method for assessing awareness was advantageous because it involved a small number of straightforward questions $(n=7)$, the questions were

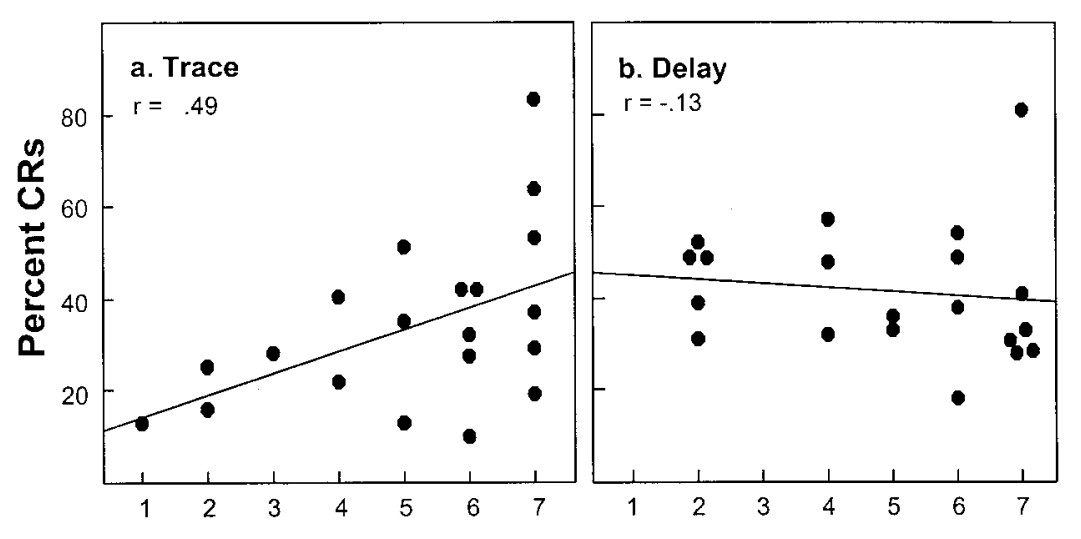

Awareness Score

Figure 1. Relationship between the awareness score obtained after the first 10 trials and the strength of conditioning_-percent conditioned responses (CRs)_across all 120 conditioning trials. (a) Performance of participants who received trace conditioning. (b) Performance of participants who received delay conditioning. 
not preceded by unrelated questions, and the questions were asked early in the conditioning session. We take the new data for singlecue delay conditioning as providing particularly strong evidence for a dissociation between awareness and human eyeblink conditioning.

It is not the case that the questions used to assess awareness were insensitive to knowledge about the relationship between the CS and the US, because in trace conditioning, a clear relationship emerged between conditioning performance and the number of questions that could be answered correctly. Accordingly, it seems reasonable to conclude that knowledge about the stimulus contingencies was irrelevant in the case of delay eyeblink conditioning. This same conclusion was reached in earlier studies of human delay eyeblink conditioning (Frcka, Beyts, Levey, \& Martin, 1983; Papka, Ivry, \& Woodruff-Pak, 1997).

In another study that compared delay and trace eyeblink conditioning (also too recent to have been considered by Lovibond \& Shanks, 2002), participants indicated on an unnumbered scale the degree to which they expected that the airpuff US would be presented on the next trial (Clark, Manns, \& Squire, 2001). The US followed the CS only $50 \%$ of the time, following a procedure introduced by Perruchet (1985). Trials were arranged such that strings of one, two, three, or four CS-alone trials were intermixed with strings of one, two, three, or four CS-US trials. In this circumstance, participants exhibit a phenomenon known as the gambler's fallacy. That is, on trials that were preceded by a string of CS-US trials, participants indicated an expectation that the US would not be presented. Conversely, on trials that were preceded by a string of CS-alone trials, participants indicated an expectation that the US would be presented. Participants exhibited this phenomenon whether they were given delay conditioning or trace conditioning.

The interesting finding was that the two groups were strikingly different with respect to the pattern of CRs that were emitted. For the participants given trace conditioning, conditioning closely paralleled the ratings of expectancy. That is, participants emitted more conditioned responses after a string of CS-alone trials (when expectancy of the US was high) than after a string of CS-US trials (when expectancy of the US was low). In contrast, for the participants given delay conditioning, conditioning closely paralleled the recent strength of the association between the CS and the US (confirming the findings of Perruchet, 1985). That is, participants emitted more conditioned responses after a string of CS-US trials than after a string of CS-alone trials. Thus, in the case of delay conditioning, CRs were most likely to be observed on those trials when participants believed most strongly that the US would not be presented.

The point is that, for participants given delay eyeblink conditioning, expectation of the US played no role in the expression of CRs. Instead, the tendency to emit a CR was influenced by the recent strength of the CS-US association. Lovibond and Shanks (2002) considered the original study by Perruchet (1985), which concerned only delay eyeblink conditioning, to provide the strongest evidence to date for a dissociation between conditioning and awareness. Our more recent study (Clark et al., 2001) provides a replication of Perruchet's finding as well as a contrast between the findings for delay and trace eyeblink conditioning.

The role of awareness in delay eyeblink conditioning has also been explored in the case of differential eyeblink conditioning
(Clark \& Squire, 1998, 1999). The positive conditioned stimulus $(\mathrm{CS}+)$ was a $1,000-\mathrm{Hz}$ tone and the negative conditioned stimulus (CS-) was white noise (or vice versa for half the participants). Participants were given a questionnaire that included 17 true or false questions about the $\mathrm{CS}+, \mathrm{CS}-$, and US after 120 trials of either differential delay eyeblink conditioning (with either a 700 -ms or 1,250-ms interstimulus interval) or differential trace eyeblink conditioning (with either a 750-ms or 1,250-ms interstimulus interval). For the two groups that received delay conditioning, the participants designated as aware conditioned no better than those who were designated as unaware. Moreover, the number of correct responses out of 17 was uncorrelated with the percentage of differential responding $(r=-.10$ and $r=+.16$ for the two delay groups). In contrast, for the two groups that received trace conditioning, knowledge about the stimulus contingencies was well correlated with the percentage of differential responding ( $r=.74$ and $r=.69$ for the two trace groups, $p \mathrm{~s}<.01$ ).

In the same study, 4 amnesic patients with bilateral damage to the hippocampal formation acquired differential delay conditioning as well as the healthy volunteers but did not as a group score above chance on the questionnaire. Further, in response to a question raised by Lovibond and Shanks (2002), we note that the 2 patients who scored the poorest on the questionnaire $(M=8.5$ correct) conditioned even better than the 2 patients who scored the best ( $M=14.0$ correct; $24.2 \%$ differential CRs across the final 60 trials vs. $13.7 \%$ differential CRs, respectively).

The finding that amnesic patients can acquire delay eyeblink conditioning without knowledge about what has occurred was first reported by Weiskrantz and Warrington (1979), and the spared capacity for acquisition and retention of delay eyeblink conditioning in amnesia has been observed repeatedly (Daum \& Ackerman, 1994; Daum, Channon, \& Canavan, 1989; Daum, Channon, \& Gray, 1992; Gabrieli et al., 1995; Schugens \& Daum, 1999).

Our recent study of differential eyeblink conditioning in amnesic patients and healthy volunteers (Clark \& Squire, 1998) was discussed at length by Lovibond and Shanks (2002). They raised several concerns about the study, including that the cutoff score ( $\geq 13$ correct out of 17 ) used to designate participants as aware may have been too high and that asking questions at the end of the session (and after other unrelated questions had been asked) was not optimal. They also remarked on what they considered to be the large number of participants who were designated unaware.

First, with respect to the matter of the cutoff score and test sensitivity, we appreciate the difficulty in evaluating a null result; that is, the finding in this study and others that knowledge of the stimulus contingencies was unrelated to delay conditioning performance (Clark \& Squire, 1998, 1999; Manns et al., 2001; Papka et al., 1997). In several studies, however, the negative finding that knowledge of the stimulus contingencies was unrelated to delay conditioning occurred together with the positive finding that the same questions revealed a strong relationship between knowledge of stimulus contingencies and trace conditioning (Clark \& Squire, 1998, 1999; Manns et al., 2001). Thus, the questions that were used were sufficiently sensitive to detect a relationship between awareness and conditioning. We have also replotted in Figure 2 the data from Clark and Squire (1998), using a stricter criterion for designating participants as unaware (i.e., $\leq 9$ questions were answered correctly out of 17). The results are the same as in the 


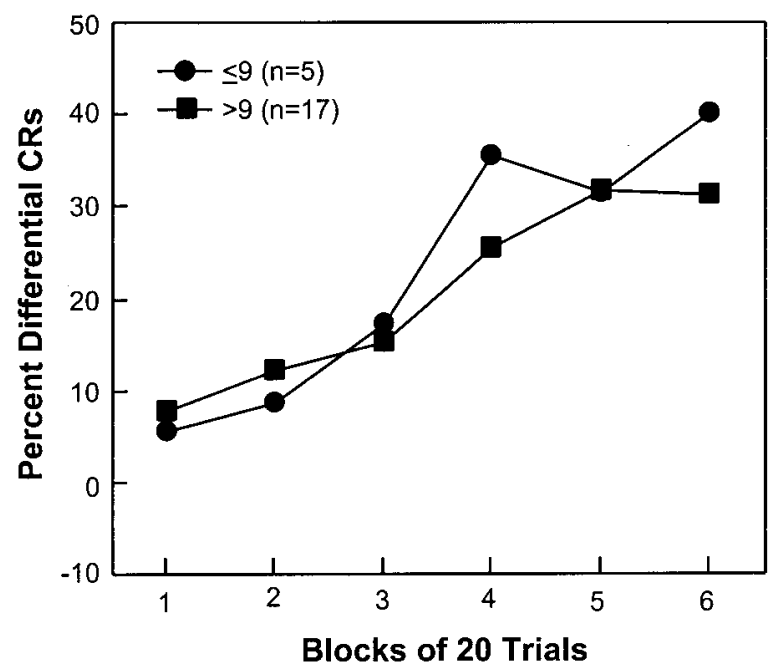

Figure 2. Performance during differential delay conditioning $(\mathrm{CS}+=$ $1-\mathrm{kHz}$ tone; $\mathrm{CS}-=$ white noise; or vice versa for half the group) as a function of awareness of the temporal relationships between the CS+, the $\mathrm{CS}-$, and the unconditioned stimulus. Participants who scored $>9$ correct on 17 true or false questions were designated aware, and participants who scored $\leq 9$ correct were designated unaware. The data are from Clark and Squire (1998), combining Figures 2A and 2B and using a stricter score ( $\leq$ 9 correct rather than $\leq 12$ correct) to designate participants as unaware. $\mathrm{CRs}=$ conditioned responses; $\mathrm{CS}+=$ positive conditioned stimulus; $\mathrm{CS}-=$ negative conditioned stimulus.

original study. Specifically, participants designated unaware conditioned as well as those designated aware.

Second, it seems likely that the age of our participants (mean age $=66.9$ years in Clark \& Squire, 1998) contributed to the relatively large number of individuals (26 of 48) who scored poorly on the knowledge test at the end of the session ( $\leq 12$ correct out of 17). Indeed, pilot studies in our laboratory with young adults suggest that most young adults become fully aware of the stimulus contingencies by the end of a typical conditioning session. We initially tested older adults in our study of differential eyeblink conditioning to provide age-matched controls for the amnesic patients (Clark \& Squire, 1998). It subsequently became clear that older adults, exactly because they do vary in how much knowledge they acquire during a conditioning session, are a useful group for studying the role of awareness in eyeblink conditioning.

Third, although many of the points raised by Lovibond and Shanks (2002) concerning the study of differential conditioning are useful considerations, the points they raised do not explain why trace eyeblink conditioning but not delay eyeblink conditioning reveals a role for awareness. The authors offered the speculation that perhaps learning the differential rule is easier in delay conditioning, whereas learning the temporal order of the CS+ and US is easier in trace conditioning. This idea appears incorrect because in the study discussed earlier (Manns et al., 2001), participants given single-cue delay conditioning and participants given singlecue trace conditioning acquired the same amount of knowledge about the CS-US relationship during the course of the conditioning session. That is, scores on the 7-item questionnaire were virtually the same for the two groups after 10 trials of conditioning, after 60 trials, and after all 120 trials. In addition, for participants who were designated as aware, conditioning performance proceeded at approximately the same rate in delay and trace conditioning (compare Figure 2A and 2B, Manns et al., 2001; also compare Figure $3 \mathrm{~A}$ and $3 \mathrm{~B}$ and Figure $3 \mathrm{C}$ and 3D, Clark \& Squire, 1998).

The findings from a different study are also of interest. Clark and Squire (1999) assessed differential delay conditioning in 12 participants who were engaged in an attention-demanding, digitmonitoring task. The group that was distracted acquired conditioning at about the same rate as 10 participants who were tested in the same way but without distraction. Lovibond and Shanks (2002) correctly pointed out that the participants who were distracted did obtain an above-chance score on the questionnaire (12.8 correct out of 17). Accordingly, it cannot be said that this group was unaware of the stimulus contingencies. However, in response to a question raised by Lovibond and Shanks, we note that the 5 individuals who scored the poorest on the questionnaire $(M=10.4$ correct) in fact conditioned a little better than the 7 who scored the best $(M=14.4$ correct; $23.2 \%$ differential CRs across the final 60 trials vs. $20.8 \%$ differential CRs). Furthermore, it is worth noting that the group that was distracted conditioned just as well as participants who were designated aware in our earlier study (Clark \& Squire, 1998, Figure 3B) and who obtained a score of 15.1 correct out of 17 on the questionnaire (16.8\% differential CRs vs. $16.1 \%$ differential CRs for distraction vs. no distraction), $t(18)=0.86, p>.10$. Thus, a significant reduction in knowledge about the stimulus contingencies, as the result of the distraction task, (15.1 to 12.8$) ; t(18)=2.48, p<.05$, had no effect on differential conditioning.

Lovibond and Shanks (2002) cited four earlier studies of differential delay eyeblink conditioning in humans, which they took as evidence for the importance of awareness and also as evidence that our findings (specifically, Clark and Squire, 1998) are discordant with the prior literature. Importantly, three of the cited studies involved nonstandard protocols in which the conditioned stimuli were words or phrases that differed in complex ways, such as grammaticality (Baer \& Fuhrer, 1982; Perry, Grant, \& Schwartz, 1977), or they differed in the configuration of color words and colors in which the words were printed (Benish \& Grant, 1980). In these instances, the phylogenetically early structures that can ordinarily support standard delay eyeblink conditioning would be unable to discriminate the $\mathrm{CS}+$ from the $\mathrm{CS}-$, and conditioning would not proceed unless participants can decipher what distinguishes the $\mathrm{CS}+$ and $\mathrm{CS}-$.

The fourth study used two different tones as CS+ and CS(Nelson \& Ross, 1974), which may not have been sufficiently salient or sufficiently easy to discriminate (also see Ross \& Nelson, 1973). Indeed, in a different study (Norman, Villablanca, Brown, Schwafel, \& Buchwald, 1974), neither control nor hemispherectomized cats were able to acquire differential conditioning using the same tones $(800 \mathrm{~Hz}$ and $2,100 \mathrm{~Hz}$ ) that were used by Nelson and Ross (1974; and also by Ross \& Nelson, 1973). Interestingly, both control and hemispherectomized cats were able to acquire differential conditioning when a different pair of tones was used $(100 \mathrm{~Hz}$ vs. $1,000 \mathrm{~Hz}$ or $200 \mathrm{~Hz}$ vs. 3,000 Hz) or when the $\mathrm{CS}+$ and $\mathrm{CS}-$ were a tone and white noise. Thus, the tone pairs used by Nelson and Ross (1974) were likely more difficult to discriminate than the tone and white noise that we used (Clark \& Squire, 1998). It is interesting to note that the conditions under 
which delay differential conditioning is independent of awareness may be more restricted than one might have suspected. It should be clear that all our studies involved a tone CS + and a white noise $\mathrm{CS}-$ (or vice versa) in the case of differential conditioning and a tone $\mathrm{CS}$ in the case of single-cue conditioning. The finding that awareness is irrelevant to delay conditioning applies only to such standard paradigms in which the CS (and the CS-) can be processed by the brain stem and related cerebellar circuitry.

A few studies have found that attention-demanding secondary tasks can sometimes retard differential delay conditioning (Carrillo, Gabrieli, \& Disterhoft, 2000; Nelson \& Ross, 1974; Ross \& Nelson, 1973). Note, however, that two tones were used as CS+ and CS - in all these studies. In one study (Ross \& Nelson, 1973) unaware participants in some groups exhibited substantial differential responding, and in two of the studies (Nelson \& Ross, 1974; Ross \& Nelson, 1973), other task factors in addition to awareness were considered to be important for conditioning. Finally, in one study (Carrillo et al., 2000) performing a verbal shadowing task reduced differential delay conditioning and also reduced the number of aware participants. However, awareness itself was unrelated to conditioning performance.

In a more recent study of differential delay conditioning in which a tone $(1000 \mathrm{~Hz})$ and static noise were used, the success of differential delay conditioning did correlate with awareness (Knuttinen, Power, Preston, \& Disterhoft, 2001). One important issue concerns the difficulty in identifying and excluding voluntary responses (Coleman \& Webster, 1988). If voluntary responses are scored as true CRs, an artificial link could be observed between awareness and apparent conditioning (for further discussion, see Manns et al., 2001). One does not know that voluntary responses were a factor in this recent study (Knuttinen et al., 2001). However, according to the criteria for excluding voluntary responses that we used in our studies (early onset, large amplitude, long duration, no effect of the airpuff US), responses resembling the average responses shown in Knuttinen et al.'s (2001) Figure 3 would have been excluded.

In summary, it appears that increasing the difficulty of differential delay eyeblink conditioning makes it more likely that awareness is important. Indeed, if the stimuli used in differential conditioning require a complex discrimination that cannot be supported by the brain stem and cerebellum, differential conditioning will likely be aided by attention, awareness, and other cognitive factors. Nevertheless, the available data, including data from two new studies not available to Lovibond and Shanks (2002), provide strong evidence that delay eyeblink classical conditioning can be acquired independently of knowledge about the stimulus contingencies. This conclusion applies to single-cue delay conditioning when a tone is used as CS and to differential delay conditioning when the $\mathrm{CS}+$ and $\mathrm{CS}-$ are a tone and white noise. Such a conclusion should not be surprising considering that indeed standard delay eyeblink conditioning can be acquired and retained independently of the forebrain.

\section{References}

Baer, P. E., \& Fuhrer, M. J. (1982). Cognitive factors in the concurrent differential conditioning of eyelid and skin conductance responses. Memory \& Cognition, 10, 81-88.

Benish, W. A., \& Grant, D. A. (1980). Subject awareness in differential classical eyelid conditioning. Bulletin of the Psychonomic Society, 15, 431-432.

Berger, T., \& Orr, W. B. (1983). Hippocampectomy selectively disrupts discrimination reversal conditioning in the rabbit nicitating membrane response. Behavioral Brain Research, 8, 49-68.

Carrillo, M. C., Gabrieli, J. D. E., \& Disterhoft, J. F. (2000). Selective effects of division of attention on discrimination conditioning. Psychobiology, 28, 293-302.

Coleman, S. R., \& Webster, S. (1988). The problem of volition and the conditioned reflex: Part II. Voluntary-responding subjects, 1951-1980. Behaviorism, 16, 17-49.

Clark, R. E., Manns, J. R., \& Squire, L. R. (2001). Trace and delay eyeblink conditioning: Contrasting phenomena of declarative and nondeclarative memory. Psychological Science, 12, 304-308.

Clark, R. E., \& Squire, L. R. (1998, April). Classical conditioning and brain systems: The role of awareness. Science, 280, 77-81.

Clark, R. E., \& Squire, L. R. (1999). Human eyeblink classical conditioning: Effects of manipulating awareness of the stimulus contingencies. Psychological Science, 10, 14-18.

Daum, I., \& Ackerman, H. (1994). Dissociation of declarative and nondeclarative memory after bilateral thalamic lesions: A case report. International Journal of Neuroscience, 75, 153-165.

Daum, I., Channon, S., \& Canavan, A. G. (1989). Classical conditioning in patients with severe memory problems. Journal of Neurology, Neurosurgery, and Psychiatry, 52, 47-51.

Daum, I., Channon, S., \& Gray, J. A. (1992). Classical conditioning after temporal lobe lesions in man: Sparing of simple discrimination and extinction. Behavioural Brain Research, 52, 159-165.

Daum, I., Schugens, M. M., Ackermann, H., Lutzenberger, W., Dichgans, J., \& Birbaumer, N. (1993). Classical conditioning after cerebellar lesions in humans. Behavioral Neuroscience, 107, 748-756.

Frcka, G., Beyts, J., Levey, A. B., \& Martin, I. (1983). The role of awareness in human conditioning. Pavlovian Journal of Biological Sciences, 18, 69-76.

Gabrieli, J. D., McGlinchey-Berroth, R., Carrillo, M. C., Gluck, M. A., Cermak, L. S., \& Disterhoft, J. F. (1995). Intact delay-eyeblink classical conditioning in amnesia. Behavioral Neuroscience, 109, 819-827.

Knuttinen, M.-G., Power, J. M., Preston, A. R., \& Disterhoft, J. F. (2001). Awareness in classical differential eyeblink conditioning in young and aging humans. Behavioral Neuroscience, 115, 747-757.

Lovibond, P. F., \& Shanks, D. R. (2002). The role of awareness in Pavlovian conditioning: Empirical evidence and theoretical implications. Journal of Experimental Psychology: Animal Behavior Processes, 28, 3-26.

Manns, J. R., Clark, R. E., \& Squire, L. R. (2000a). Awareness predicts the magnitude of single-cue trace eyeblink conditioning. Hippocampus, 10, $181-186$.

Manns, J. R., Clark, R. E., \& Squire, L. R. (2000b). Parallel acquisition of awareness and trace eyeblink conditioning. Learning \& Memory, 7 , 267-272.

Manns, J. R., Clark, R. E., \& Squire, L. R. (2001). Single-cue delay eyeblink classical conditioning is unrelated to awareness. Cognitive, Affective, and Behavioral Neuroscience, 1, 192-198.

Mauk, M. D., \& Thompson, R. F. (1987). Retention of classically conditioned eyelid responses following acute decerebration. Brain Research, 403, 89-95.

McGlinchey-Berroth, R., Carrillo, M. C., Gabrieli, J. D. E., Brawn, C. M., \& Disterhoft, J. F. (1997). Impaired trace eyeblink conditioning in bilateral, medial-temporal lobe amnesia. Behavioral Neuroscience, 100, 243-252.

Nelson, M. N., \& Ross, L. E. (1974). Effects of masking tasks on differential eyelid conditioning: A distinction between knowledge of stimulus contingencies and attentional or cognitive activities involving them. Journal of Experimental Psychology, 102, 1-9. 
Norman, R. J., Villablanca, J. R., Brown, K. A., Schwafel, J. A., \& Buchwald, J. S. (1974). Classical eyeblink conditioning in the bilaterally hemispherectomized cat. Experimental Neurology, 44, 363-380.

Papka, M., Ivry, R. B., \& Woodruff-Pak, D. S. (1997). Eyeblink classical conditioning and awareness revisited. Psychological Science, 8, 404-408.

Perruchet, P. (1985). A pitfall for the expectancy theory of human eyelid conditioning. Pavlovian Journal of Biological Sciences, 20, 163-170.

Perry, L. C., Grant, D. A., \& Schwartz, M. (1977). Effects of noun imagery and awareness of the discriminative cue upon differential eyelid conditioning to grammatical and ungrammatical phrases. Memory \& Cognition, 5, 423-429.

Ross, L. E., \& Nelson, M. N. (1973). The role of awareness in differential conditioning. Psychophysiology, 10, 91-94.

Schmaltz, L. W., \& Theios, J. (1972). Acquisition and extinction of a classically conditioned response in hippocampectomized rabbits (Oryctolagus cuniculus). Journal of Comparative and Physiological Psychology, 79, 328-333.

Schugens, M. M., \& Daum, I. (1999). Long-term retention of classical eyeblink conditioning in amnesia. Neuroreport, 10, 149-152.
Solomon, P. R., \& Moore, J. W. (1975). Latent inhibition and stimulus generalization of the classically conditioned nicitating membrane response in rabbits (Oryctolagus cuniculus) following dorsal hippocampal ablation. Journal of Comparative and Physiological Psychology, 89, 1192-1203.

Steinmetz, J. E., Lavond, D. G., Ivkovich, D., Logan, C. G., \& Thompson, R. F. (1992). Disruption of classical eyelid conditioning after cerebellar lesions: Damage to a memory trace system or a simple performance deficit? Journal of Neuroscience, 12, 4403-4426.

Thompson, R. F., \& Krupa, D. J. (1994). Organization of memory traces in the mammalian brain. Annual Review of Neuroscience, 17, 519-549.

Topka, H., Valls-Solé, J., Massaquoi, S. G., \& Hallett, M. (1993). Deficit in classical conditioning in patients with cerebellar degeneration. Brain, 116, 961-969.

Weiskrantz, L., \& Warrington, E. K. (1979). Conditioning in amnesic patients. Neuropsychologia, 17, 187-194.

Received July 13, 2001

Accepted July 19, 2001

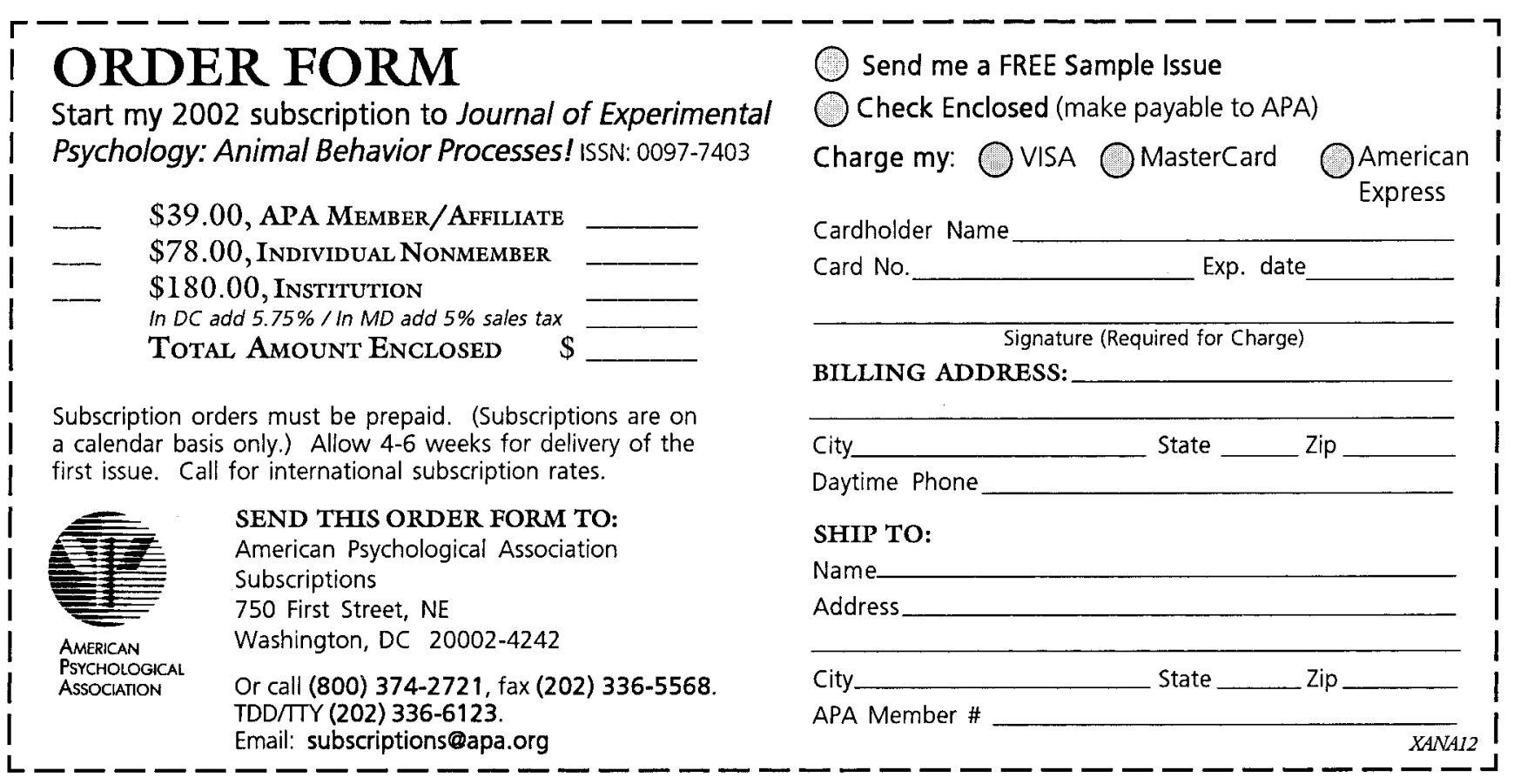

